\title{
Multi-Frequency VLBA+Effelsberg Observations of 1038+528 A/B
}

\section{J. Rioja}

Joint Institute for VLBI in Europe, Dwingeloo, Netherlands

R. W. Porcas

Max-Planck-Institut für Radioastronomie, Bonn, Germany

Abstract. We made a November 1995 observation of the close ( 33 arc sec) pair $1038+528$ A,B using the VLBA+Effelsberg at 2.3 and $8.4 \mathrm{GHz}$, and the VLBA alone at $15 \mathrm{GHz}$. Our analysis of the precise astrometric separation between the core peak in $\mathrm{A}$ and a component $\sim 1.8$ mas from the core in $B$ sheds new light on the observed changes with time and frequency.

\section{Temporal Changes}

The change in angular separation between quasars $\mathrm{A}$ and $\mathrm{B}$ at $8.4 \mathrm{GHz}$ in 1995 continues the trend of previous epochs (see Fig. 1a), and confirms that an outward motion of the B reference feature is the dominant contribution (Rioja 1997). We have used various methods to better quantify this, now that we have a longer time baseline: (a) a direct estimate of the separation rate between the core and reference-component from the $\mathrm{B}$ quasar hybrid maps; this gives a value of $12.8 \mu \mathrm{as} / \mathrm{yr}$ (see Fig. 1b). (b) a decomposition of the A-B changes along two preferred PAs in the sources ( $\mathrm{PA}-53^{\circ}$ in $\mathrm{B} ; 0-35^{\circ}$ in $\mathrm{A}$ ), with a linear fit to the changes deduced in $\mathrm{B}$. The best fit, with $\mathrm{PA}=25^{\circ}$ in quasar $\mathrm{A}$, corresponds to a $\mathrm{B}$ reference component motion of $16.6 \mu \mathrm{as} / \mathrm{yr}$; for the measured inner PA in $\mathrm{A}$ of $15^{\circ}$ it is $15.6 \mu \mathrm{as} / \mathrm{yr}$. All these values correspond to sub- or superluminal velocities, depending critically on the choice of the cosmological parameters $\mathrm{H}_{o}$ and $q_{0}$. The difference between the estimates from (a) and (b), if real, would correspond to an outward motion of the B core of ca. $3 \mu \mathrm{as} / \mathrm{yr}$.

a)

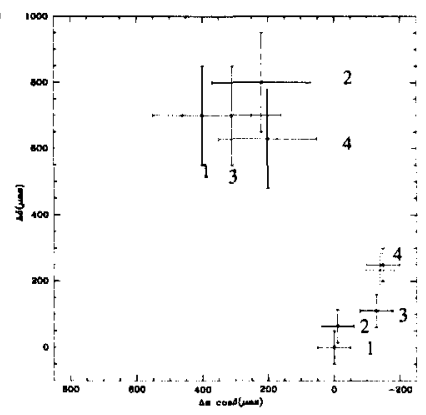

b)

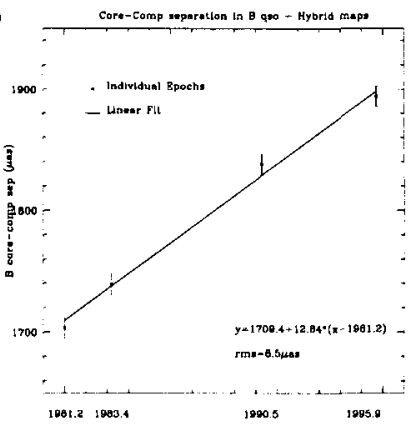

Figure 1. a) Relative position measurements between $1038+528 \mathrm{~A}$,B. Large crosses $=2.3 \mathrm{GHz}$, small crosses $=8.4 \mathrm{GHz}$ and hatched cross $=15 \mathrm{GHz}$. Numbers indicate epoch. Origin corresponds to $8.4 \mathrm{GHz}$ separation at epoch 1 . b) Linear fit to changes in core/ref-component separation in maps of quasar $\mathrm{B}$ at $8.4 \mathrm{GHz}$ 


\section{Frequency Registration}

As in previous epochs, the separation between the $\mathrm{A}$ and $\mathrm{B}$ reference features differs significantly between 2.3 and $8.4 \mathrm{GHz}$, with an offset from 500 to $700 \mu$ as (Fig. 1a). Our measurement at the new frequency of $15 \mathrm{GHz}$, however, gives a separation essentially identical to that at $8.4 \mathrm{GHz}$. Marcaide \& Shapiro (1984) ascribe all the $2.3 / 8.4 \mathrm{GHz}$ offset to quasar $\mathrm{A}$, and a wavelength-dependent position shift of the "core" proportional to $\lambda^{\beta}$, with acceptable values of $\beta$ between 0.7 and 2 . Our new results at $2.3,8.4$ and $15 \mathrm{GHz}$ are compatible with a new lower bound for $\beta$ close to 1 , and an infinite upper bound. However, at both 8.4 and $15 \mathrm{GHz}$ the maps of $\mathrm{A}$ reveal very similar core-jet morphology (see Fig. 2), with a prominent knot, k1, offset from the core by an amount remarkably close to the observed $2.3 / 8.4 \mathrm{GHz}$ offset. These two components are blended together at $2.3 \mathrm{GHz}$ by the $\sim 4$ times larger beamwidth, so that at least part of the offset is a natural result of this blending at $2.3 \mathrm{GHz}$, enhanced by the different spectral indices of the core and $\mathrm{k} 1$ components. Moreover, the relative position of a second, weak knot, $\mathrm{k} 2$, and $\mathrm{k} 1$, seen at $8.4 \mathrm{GHz}$, is very similar to that between the "core" and first knot feature seen at $2.3 \mathrm{GHz}$ (Fig. 2 ). We propose a new registration based on the identification of these features. This implies the existence of an offset of $\sim 250 \mu$ as between the 2.3 and $8.4 \mathrm{GHz}$ positions of the $\mathrm{B}$ reference features.
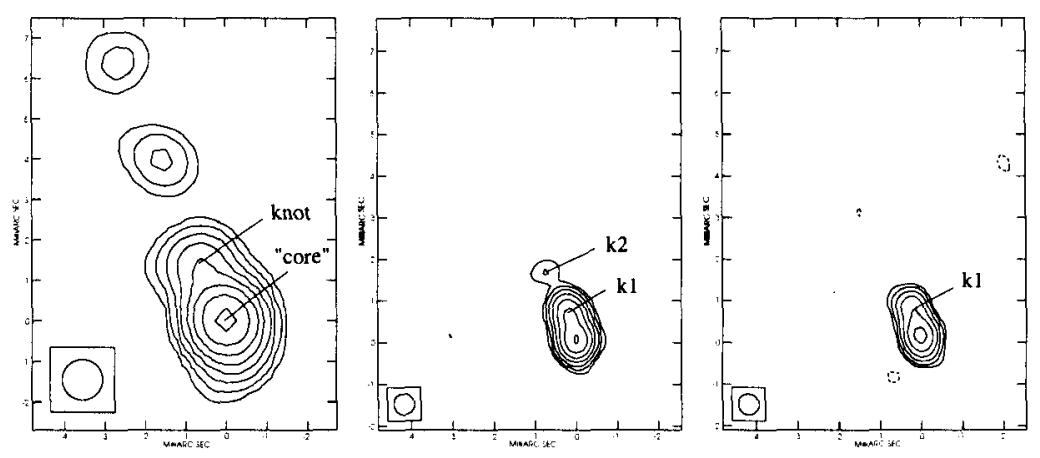

Figure 2. Super-resolved map of $1038+528 \mathrm{~A}$ at $2.3 \mathrm{GHz}$ (left) and normalresolution maps at $8.4 \mathrm{GHz}$ (center) and $15 \mathrm{GHz}$ (right).

Acknowledgments. We thank D. Graham and A. Beasley for assistance with the observations. M. Rioja acknowledges support by the EU under contract CHGECT920011. The National Radio Astronomy Observatory is a facility of the National Science Foundation, operated under a cooperative agreement by Associated Universities, Inc.

\section{References}

Marcaide, J. M., \& Shapiro, I. I. 1984. ApJ, 276, 56-59.

Rioja, M. J., et al. 1997. $A \& A, \mathbf{3 2 5}, 383-390$. 